\title{
Comparison of the haematoxylin basic fuchsin picric acid method and the fluorescence of haematoxylin and eosin stained sections for the identification of early myocardial infarction
}

\author{
HK AL-RUFAIE, RA FLORIO, EGJ OLSEN \\ From the National Heart Hospital, Westmoreland Street, London WIM 8BA
}

SUMMARY A retrospective study has been carried out on the necropsy material from 30 patients who have died after a clinically diagnosed myocardial infarction. This study has been undertaken to compare the reliability of the fluorescence of infarcted myocardium when stained by haematoxylin and eosin and an adjacent section stained by the haematoxylin basic fuchsin picric acid (HBFP) method to detect early ischaemia. The results showed that the fluorescence technique is reliable, reproducible and coincides with the findings obtained by HBFP stain.

The diagnosis of myocardial infarction of less than six hours duration by histological, histochemical or biochemical methods has always been subjective. Morphological changes in the myocardium less than six hours after the clinical event of infarction are minimal, making it impossible at light microscopic level, to assess the degree of irreversible damage that has taken place. Reviews of the histochemical investigations to demonstrate the changes in fat, glycogen, succinic dehydrogenase, cytochrome oxidase, phosphorylase, uridine diphosphate, glucose-glycogen transferase, B hydroxybutyrate dehydrogenase and isocitrate dehydrogenase have been carried out. ${ }^{12}$ These methods can be difficult to interpret. Furthermore, fresh tissue, often impossible to obtain, is essential for histochemical analysis. This is also a prerequisite for evaluation at electron microscopic level. ${ }^{3}$

Biochemical methods, which include the changes in the $\mathrm{Na}: \mathrm{K}$ ratio within the myocardium have been reported to be the most reliable techniques for the detection of early infarction. ${ }^{4}$ Other methods such as staining by acid or basic fuchsin have also been reported. ${ }^{5-7}$ These have the added advantage of permitting assessment on paraffin-embedded tissue. More recently the examination of tissue sections stained by haematoxylin and eosin by short wavelength (blue) light has also been reported.'

In the opinion of the present authors the only

Accepted for publication 6 January 1983 method which gave consistent and reproducible results in the detection of early infarction was hitherto the haematoxylin basic fuchsin picric acid (HBFP) stain. ${ }^{8}$ We have undertaken this study to compare the results obtained by this method with the fluorescence of haematoxylin and eosin stained sections.

\section{Material and methods}

A search through the necropsy records for the past three years yielded 30 cases of patients who had 3 died after a clinically diagnosed myocardial infarction, with the period of survival varying between 30 min and $36 \mathrm{~h}$. In this study of 30 cases there were 23 men and 7 women of ages ranging from 29 to $68 \mathrm{yr}$. The clinical diagnosis based upon history, physical o findings and electrocardiographic evidence in all of the cases showed myocardial infarction as the most $\mathrm{or}$ likely cause of death, no additional causes being $N$ identified at post mortem. The selection of only that material which was likely to be infarcted enabled the $\underset{O}{0}$ direct comparison of positive reactions, rather than a blind study to assess the already proven sensitivity of the two techniques. In every case the whole heart $\stackrel{\mathcal{D}}{\rightarrow}$ was available for study and transverse sections of 0 both ventricles were analysed microscopically. All $\overrightarrow{0}$ tissue had been fixed in $10 \%$ neutral buffered for- $\overrightarrow{\mathbb{D}}$ malin, it was processed and embedded for paraffin sectioning, and cut at $5 \mu \mathrm{m}$ thickness. Adjacent sections were stained by haematoxylin and eosin and HBFP stains. 

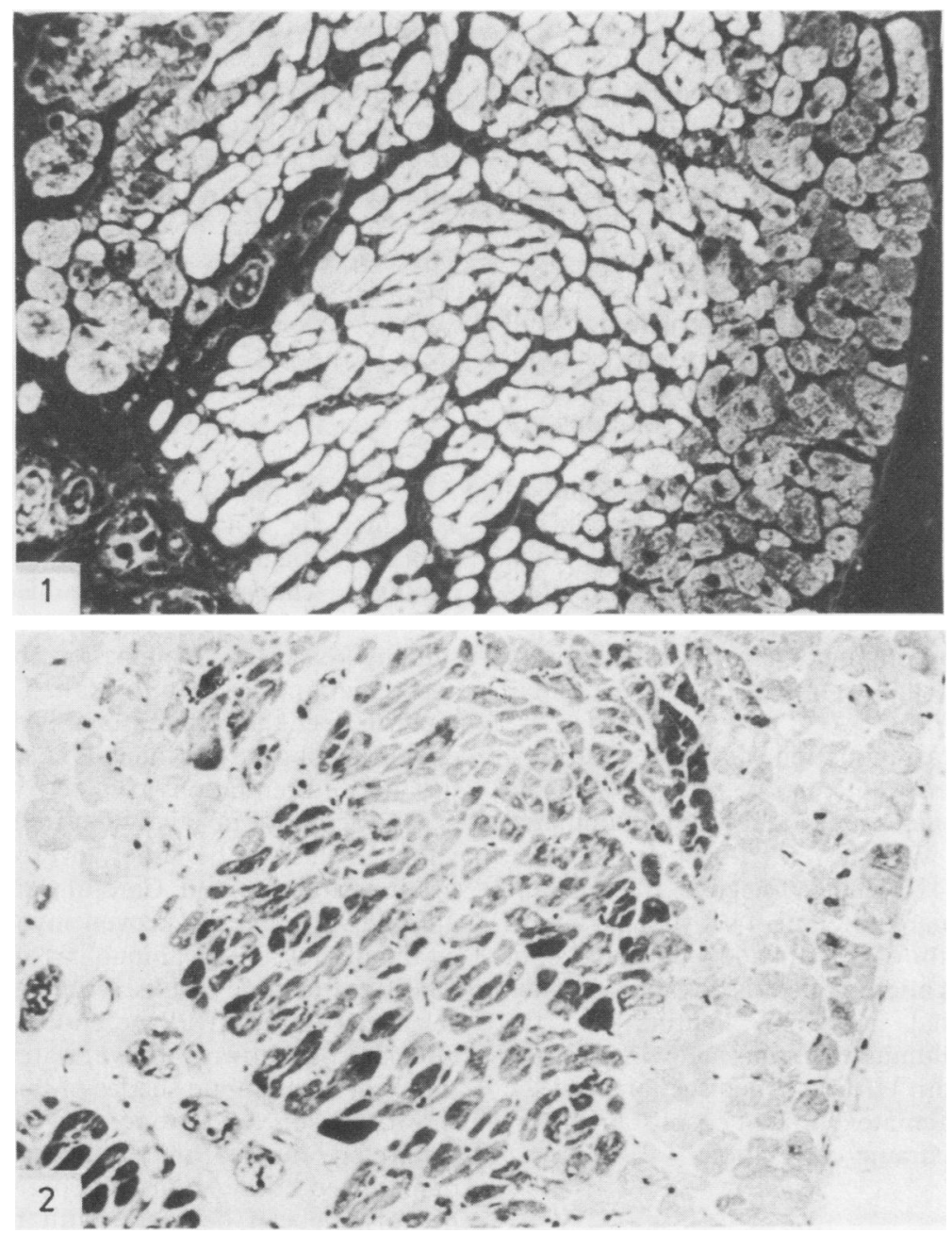

Fig. 1 Haematoxylin and eosin stained section viewed by short wavelength blue light. The infarcted area is the light, almost white area, compared to the grey superficial zone (right side of the photograph) which are the unaffected muscle fibres. Fig. 2 An adjacent section to that illustrated in Fig. 1, stained by haematoxylin basic fuchsin picric acid: the area of infarction is the dark zone. There is perfect correlation with the affected area in Fig. 1.

The full methodology of the HBFP stain is quoted here. As difficulties have been experienced with this method by some workers, we have included some notes on the staining technique which we have found useful in obtaining reliable results.

1 Dewax sections and hydrate in water.

2 Stain nuclei with an alum haematoxylin (solution A), differentiate in $1 \%$ acid alcohol and blue by washing in running tap water for $5 \mathrm{~min}$.

3 Stain in $0.1 \%$ basic fuchsin in distilled water (solution B) for $3 \mathrm{~min}$ (Note (i)).
4 Rinse slides in distilled water.

5 Rinse slides in absolute acetone.

6 Differentiate in $0.1 \%$ picric acid in absolute acetone (solution C) for 15-20 s (Note (ii)).

7 Rinse in absolute acetone.

8 Clear in fresh xylol, Inhibisol or CNP 30.

9 Mount in any synthetic resin mountant.

Ischaemic heart muscle, RBCs, collagen tissue: crimson red.

Nuclei: blue.

Other tissues and structures: yellow. 
Nòtes

(i) The only suitable basic fuchsin found by the authors is available from Raymond A Lamb, 6 Sunbeam Road, London NW12 6JL. This solution must be freshly prepared.

(ii) At the differentiation stage it is better to treat the slides individually, face up on a slide rack flooding the slide with the differentiating solution whilst gently rocking the slide for 15-20 s. Flood the slide again with fresh solution and then immediately wash in the absolute acetone. Finally wash the slide with fresh clearing agent. It is important to ensure that all solutions are fresh and uncontaminated.

Differentiation is completed when the red blood cells and collagen tissue in the section remain red whilst all other tissue components (other than ischaemic muscle) are yellow. If these parameters are not closely observed false positive or false negative results may be obtained. The decolouration of the red blood cells appears to be the most sensitive criterion.

The fluorescent examination was carried out on a Standard 14 Zeiss microscope fitted with an epifluorescence condenser model IV Fl, illuminated with a $12 \mathrm{~V} 100 \mathrm{~W}$ tungsten halogen light source and viewed with Neofluar objectives. The filters used were a primary filter of $440-490 \mathrm{~nm}$ and a dichromatic reflector of $510 \mathrm{~nm}$ and a barrier filter of $520 \mathrm{~nm}$. With this microscope it is possible to switch from conventional substage lighting to the epifluorescence illumination thus allowing for the examination of both HBFP stained sections and the fluorescence of haematoxylin and eosin stained sections with the maximum correlation.

\section{Results}

The cases were divided into two groups based upon the clinical history and on the histological changes seen in the sections. In group I there were 14 cases with evidence of less than six hours of ischaemia. Group II consisted of 16 cases with a history of longer than six hours and up to 36 hours of ischaemia. In 28 of the cases the coronary arteries showed either complete occlusion of one or more of the major vessels or moderate to severe atherosclerotic changes. In two cases no vascular abnormalities were found.

The blocks for histological analysis were taken to comprise a complete cross section of both ventricles. In this way it was possible to relate the areas of infarction to the vessel pathology. The study was carried out by first examining the haematoxylin and eosin stained sections for fluorescence and mapping the positive areas. These areas were seen as bright yellow with clear-cut cell borders (Fig. 1) or yellow or red/brown with ill-defined borders of the myocardial fibres. Analysing carefully and comparing group I with group II we found that the colour changes described above were not dependent on the duration of the ischaemia, or related to the patients age. Normally perfused myocardium was seen as dull olive green or red/brown with a clearly demarcated cell wall. The areas defined by fluorescence were then compared with the crimson red stained areas in the HBFP stained sections (Fig. 2). It was found in all cases that they coincided exactly.

\section{Discussion}

The first six hours postinfarction are the most difficult to diagnose due to the lack of visible morphological change or reliable histochemistry. The HBFP stain has been reported to give a positive staining reaction $30 \mathrm{~min}$ after coronary occlusion and remain positive up to $36 \mathrm{~h}$. This technique has hitherto been the only reasonably reliable method of $\Phi$ demonstrating early ischaemic changes. The difficul- $\vec{\bullet}$ ties of differentiation have led some workers to experience false positive or false negative staining, although this can be largely overcome if our modifications are observed. Careful examination of these 30 cases of clinically proven myocardial infarction by the fluorescent technique has also proved to give consistent reliable results, with extremely good correlation with the HBFP method. Some time needs to be spent on known positive material to appreciate the sometimes subtle shades of fluorescence such as those between yellow to red/brown with hazy border (infarcted muscle) and, red/brown with a clearly demarcated border (normally perfused muscle), but no mistake can be made with the bright yellow fluorescence when it is present. Waviness as $ᄋ$ described by Bouchardy and Majno ${ }^{9}$ was observed $₹$ in some of the cases of both groups. These areas 을 were invariably positive by both techniques. How- $\rightarrow$ ever, in other instances the wave pattern was absent in positive areas. This suggests that the change of $N$ waviness is not always reliable as an indication of ischaemia. The animal experiments of Lie et $a^{6}$ have $\stackrel{N}{\circ}$ shown that the HBFP method becomes positive $\underset{\mathrm{N}}{\mathrm{N}}$ after the first $\mathbf{3 0}$ min from occlusion. Whilst there are no animal studies to show exactly at which point 0

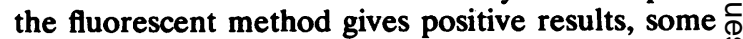
of our patients had a clinical history of no more than $\stackrel{\odot}{+}$ $30 \mathrm{~min}$ from the onset of symptoms to death. These 0 cases still gave unequivocally positive results.

It is concluded from our results that both $\stackrel{\odot}{\circ}$ haematoxylin and eosin fluorescence and the HBFP $\stackrel{\mathbb{Q}}{Q}$ stain are reliable, reproducible methods of demon- $\bar{\sigma}$ strating early myocardial infarction. 
For those workers, who dislike or mistrust the results obtained with the HBFP stain, comparison with the fluorescent method would help to form a conclusive diagnosis.

\section{References}

${ }^{1}$ Olsen EGJ. The pathology of the heart. 2nd ed. Basingstoke and London: The Macmillan Press, 1980:116-7.

${ }^{2}$ Carle Birdsall N. Autofluorescence in the identification of myocardial infarcts. Hum Pathol 1981;12:643-6.

${ }^{3}$ Caulfied J, Klionsky B. Myocardial ischemia and early infarction: an electron microscopic study. Am J Pathol 1959;35:489-523.

4 Zugibe FT, Bell P, Conley TL, Standish MS. Determination of myocardial alterations at autopsy in the absence of gross and microscopic changes. Circulation 1965;32 suppl II:35-6.
5 Poley RW, Fobes CD, Hall MJ. Fuchsinophilia in early myocardial infarction. Arch Pathol 1964;77:325-9.

${ }^{6}$ Lie JT, Holley KE, Kampa WR, Titus JL. New histochemical method for morphologic diagnosis of early stages of myocardial ischemia. Mayo Clin Proc 1971;46:319-27.

' Zugibe FT Jr, Zugibe FT. Fuchsinophilia and fuchsinorrhagia staining techniques. Arch Pathol 1973;96:243-4.

' Nayer A, Olsen EGJ. The use of the basic fuchsin stain in the recognition of early myocardial ischemia. Cardiovasc Res 1974;8:391-4.

9 Bouchardy B, Majno G. Histopathology of early myocardial infarcts. Am J Pathol 1974;74:301-30.

Requests for reprints to: Dr EGJ Olsen, National Heart Hospital, Westmoreland Street, London W1M 8BA, England. 